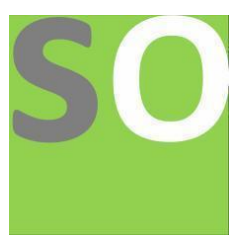

Article title: Relativistic Maxwell Theory and Einstein Field Equation derived from Variational Principle Authors: Shubham Singh[1]

Affiliations: Independent researcher[1]

Orcid ids: 0000-0003-3754-7280[1]

Contact e-mail: shubhamsingh2496@gmail.com

License information: This work has been published open access under Creative Commons Attribution License $\mathrm{http}: / / c r e a t i v e c o m m o n s . o r g / l i c e n s e s / b y / 4.0 /$, which permits unrestricted use, distribution, and reproduction in any medium, provided the original work is properly cited. Conditions, terms of use and publishing policy can be found at https://www.scienceopen.com/.

Preprint statement: This article is a preprint and has not been peer-reviewed, under consideration and submitted to ScienceOpen Preprints for open peer review.

DOI: 10.14293/S2199-1006.1.SOR-.PPDWFUG.v1

Preprint first posted online: 11 November 2021

Keywords: Varitional Principle, Maxwell's field equation, Einstein's field equation, Hilbert Action, Special relativity 


\title{
Relativistic Maxwell Theory and Einstein Field Equation derived from Variational Principle
}

\author{
Shubham Singh \\ 4/319, Lucknow, 226002,Uttar Pradesh, India \\ shubhamsingh2496@gmail.com
}

\begin{abstract}
In this article, I'll be reviewing relativistic mechanisms using the calculus of variation in the classical limit. The variational principle is considered to be one of the most important mechanisms to build a theory. Newton's second law of motion is a consequence of EulerLagrange equations which gives the least (or stationary) trajectory of a particle between any two arbitrary points. I'll the use action principle by deriving the relativistic Maxwell's field equation, geodesic equation, and Einstein's field equation.
\end{abstract}

Keywords: Varitional Principle, Maxwell's field equation, Einstein's field equation, Hilbert Action, Special relativity.

\section{Contents}

1 Introduction $\quad 3$

2 Mathematical Preliminary $\quad 3$

3 Special Relativity 3

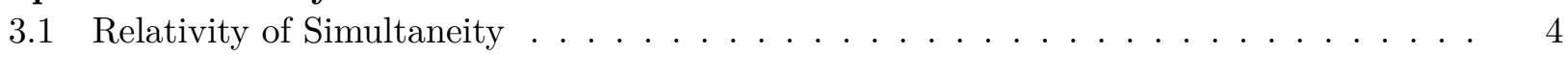

3.2 Lorentz Transformations . . . . . . . . . . . . . . . . . . . . . 4

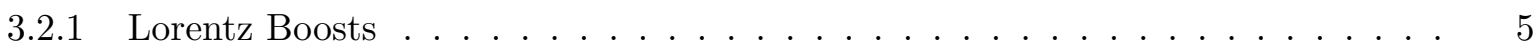

3.2 .2 Lorentz Rotation . . . . . . . . . . . . . . . . . . . . . 6

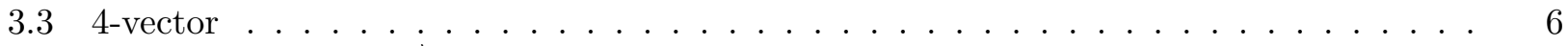

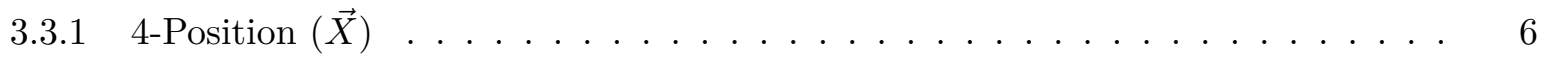

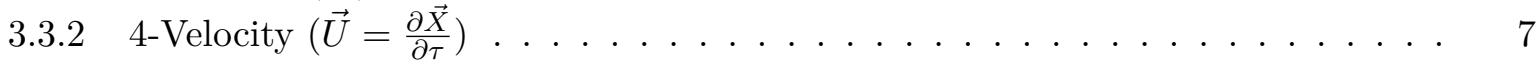

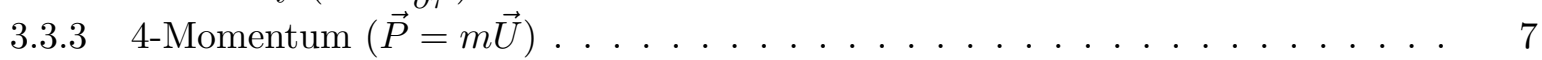

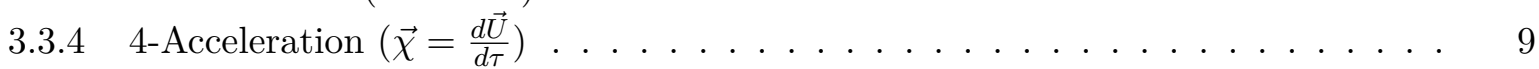

3.4 Rindler Coordinate . . . . . . . . . . . . . . . . . . . . . . 12

4 Maxwell Theory 13

4.1 Relativistic Lorentz Force . . . . . . . . . . . . . . . . . . . . . . . 13

4.2 Maxwell Equations . . . . . . . . . . . . . . . . . . . . . . . 14

5 General Relativity $\quad 16$

5.1 Equivalence Principle . . . . . . . . . . . . . . . . . . . . . . . 16

5.2 Definitions, Properties \& Theorems . . . . . . . . . . . . . . . . . 17

5.3 Variation in determinant metric $-\operatorname{det} g$ and volume element $\sqrt{-\operatorname{det} g} \ldots \ldots 17$

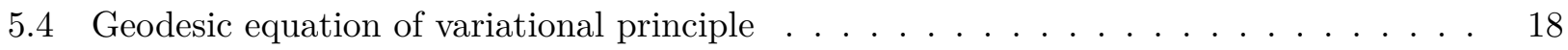

5.5 Einstein Field Equation ． . . . . . . . . . . . . . . . . . . . . . . . . . 19 


\section{Introduction}

Lagrange introduced the analytical formalism of Newtonian mechanics, which is now called the calculus of variation or Lagrangian mechanics. Legendre transformation of Lagrangian mechanics gives hamiltonian mechanics. In this article, I'll restrict to Lagrangian mechanics and deal with the topics of special relativity and general relativity at the introductory level.

In special relativity section (3), I have discussed the flat space-time geometry to understand the electrodynamic. I have used the usual Euler-Lagrange equation to derive the relativistic (covariant) Maxwell's equation.

In the general relativity section (5), I have used the variational principle to derive the geodesic equation, and then from Einstein-Hilbert action derived the Einstein field equation.

\section{Mathematical Preliminary}

Unlike Newtonian mechanics, relativity considers spacetime a single entity, i.e., three space + one time $(3+1)$ dimensional pseudo-Riemannian manifold $(\mathrm{M})$. The tangent space described on it is called Minkowski space or pseudo-Euclidean vector space. Pseudo-Riemannian manifold is a topological space that is smooth and continuously differential with a defined metric tensor such that the fundamental theorem of Riemannian geometry is satisfied. Special relativity is a special case of the general theory of relativity. It considers the speed of light to be constant in a local inertial frame. Basis vectors are constant and orthogonal throughout the Minkowski space. However, in general relativity, basis vectors may vary depending upon the intrinsic nature of the metric. Thus, in special relativity, transformation rules are linear, called Lorentz transformation, and in general relativity, these transformations are no longer valid until the metric is locally flat. Vectors and dual vectors are defined by their usual properties.

Distance $s$ given in Minsowski space between two events is straight line. $d s=\sqrt{\eta_{\mu \nu} d x^{\mu} d x^{\nu}}$. In pseudo-Riemannian manifold, metric $\eta$ can be replaced by more general metric $g$ such that $d s=\sqrt{g_{\mu \nu} d x^{\mu} d x^{\nu}}$.

Action should be invariant, hence, one could define Lagrangian by scalar function or functional. For example: Length of Faraday tensor (2-form) $F_{\mu \nu} F^{\mu \nu}$, Ricci Scalar $F$ and metric 2 -form $g$, etc are used to define the Lagrangian.

\section{Notations:}

- I'll use 4-position: $\left(X^{0}, X^{1}, X^{2}, X^{3}\right) \equiv\left(X^{t}, X^{x}, X^{y}, X^{3}\right) \equiv(t, x, y, z) \equiv X^{\mu} \equiv\left(X^{0}, X^{n}\right)$. NOTE: I"ll use Greek indices to denote spacetime $(1+3 \mathrm{D})$ components and Latin indices reserved for spatial (3D) components.

- For 4-velocity: $\left(U^{0}, U^{1}, U^{2}, U^{3}\right) \equiv\left(U^{t}, U^{x}, U^{y}, U^{3}\right) \equiv\left(\gamma v^{t}, \gamma v^{x}, \gamma v^{y}, \gamma v^{z}\right)$ where $v^{x}, v^{y}$ and $v^{z}$ is ordinary velocity and I'll use dummy variable to show time component of 4velocity,i.e., $v^{0}=v^{t}=c$. 3 -velocity is $v=v^{x}+v^{y}+v^{z} . \dot{x}=\frac{\partial x}{\partial t}$, that is, derivative with respect to coordinate time.

\section{$3 \quad$ Special Relativity}

Postulates:

1. Universal Constant: Speed of light $c$ (in a free space) is constant in all local inertial frame independent of the observers.

2. Galilean Invariance: Laws of motions are independent of the frame of reference. This implies physics is same in all reference frame. 
Minkowshi space has metric signature $(+--)$ or $(-+++)$ depending upon the time-like or space-like trajectory. Explicitly $\operatorname{sign}(-+++)$ is

$$
\eta_{\mu \nu}=\left[\begin{array}{cccc}
-1 & 0 & 0 & 0 \\
0 & 1 & 0 & 0 \\
0 & 0 & 1 & 0 \\
0 & 0 & 0 & 1
\end{array}\right]
$$

Spacetime invariance: The quantity upon which every observer will agree is called spacetime invariance. For example, all the observers must agree on the distance between any two events in spacetime. This is called proper length $s$. Similarly, for timelike trajectories, there is the proper time $(\tau)$.

Explicitly proper time is given as

$$
(\Delta \tau)^{2}=(\Delta t)^{2}-(\Delta x)^{2}-(\Delta y)^{2}-(\Delta z)^{2}
$$

and proper length as

$$
(\Delta s)^{2}=-(\Delta t)^{2}+(\Delta x)^{2}+(\Delta y)^{2}+(\Delta z)^{2}
$$

Proper length and proper time are related as

$$
(\Delta s)^{2}=-c^{2}(\Delta \tau)^{2}
$$

Equation (1) can also be written as

$$
d \tau=\sqrt{\eta_{\mu \nu} d x^{\mu} d x^{\nu}}
$$

Euler-Lagrange equation for Lagrangian $\mathcal{L}$ is defined as

$$
\frac{d}{d X^{\nu}} \frac{\partial \mathcal{L}}{\partial\left(\partial_{\nu} X^{\mu}\right)}=\frac{\partial \mathcal{L}}{\partial X^{\mu}}
$$

\subsection{Relativity of Simultaneity}

I'll describe Simultaneity using a thought experiment. Two events are synchronised means the time taken by a light ray from $\mathrm{A}$ to $\mathrm{B}$ is the same as from $\mathrm{B}$ to $\mathrm{A}$. If two events happened at the time (by the synchronised clock) then those events are simultaneous. $t_{A}-t_{B}=t_{A}^{\prime}-t_{B} \Longrightarrow$ simultaneous. And relativity suggests that for the different observers (different inertial frames) simultaneity is not absolute. For example, 1 suppose you are on a moving train with your friend sitting across from you (you are facing the direction of motion), she suddenly turns on the flashlight on your face, the light will take time $t$ to reach you, and someone observing from the platform (rest frame) will measure the shorter time $t^{\prime}$ for the light to reach you. It is because of motion of the train will reduce the distance for the light in the rest-frame relative to the moving frame as for the rest observer, speed of light is $c . t>t^{\prime}$.

Causality is a consequence of simultaneity, which states that cause can not proceed event. In other words, you can't listen to the sound of the bomb before its explosion. Thus super luminosity is prohibited.

\subsection{Lorentz Transformations}

$$
\eta_{\mu \nu} L_{\rho}^{\mu} L_{\sigma}^{\nu}=\eta_{\rho \sigma}
$$

Let's take two coordinates $\mathrm{y}$ and $\mathrm{x}$, and transform $\mathrm{x}$ to get $\mathrm{y}$. 


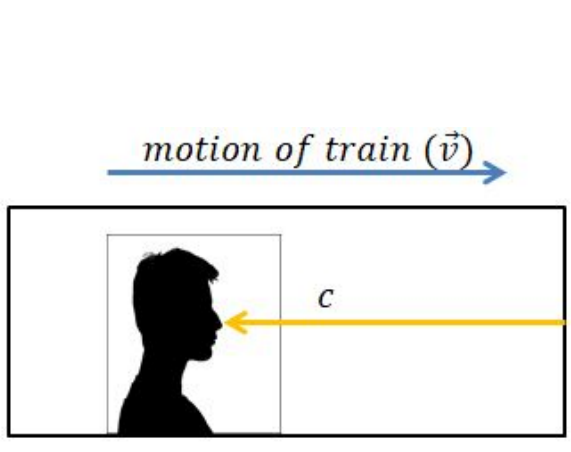

(a) Rest frame (inside of train)

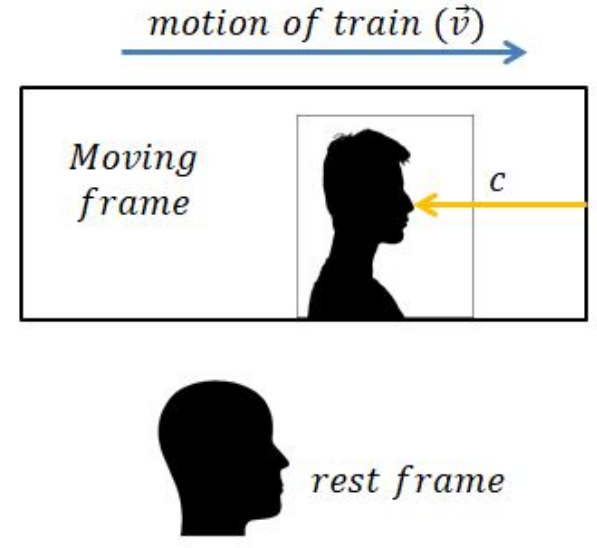

(b) Rest frame relative to moving frame

Figure 1: (1a) An observer inside of the train is at rest with respect to its surrounding. (1b) However, an observer outside the train (at rest) will observe the train and you as a moving frame. This relativity motion and for both observers inside the train and outside will measure the same speed of light, due to which events will no longer be simultaneous of both observers.

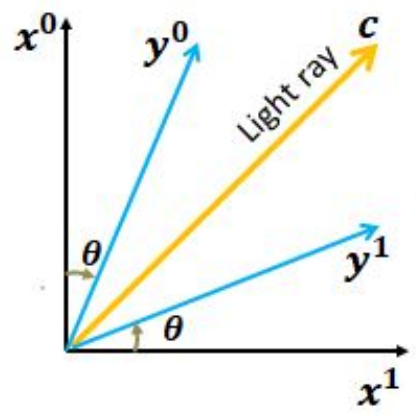

Figure 2: Lorentz boost is similar to hyperbolic rotation by $\theta$

\subsubsection{Lorentz Boosts}

Boosts, in simple words, can be defined as transnational motion.

$$
\left[\begin{array}{l}
y^{0} \\
y^{1} \\
y^{2} \\
y^{3}
\end{array}\right]=\left[\begin{array}{cccc}
\cosh \theta & -\sinh \theta & 0 & 0 \\
-\sinh \theta & \cosh \theta & 0 & 0 \\
0 & 0 & 1 & 0 \\
0 & 0 & 0 & 1
\end{array}\right]\left[\begin{array}{l}
x^{0} \\
x^{1} \\
x^{2} \\
x^{3}
\end{array}\right]
$$

If $\cosh \theta=\frac{1}{\sqrt{1-\left(\frac{v}{c}\right)^{2}}} \& \cosh \theta=\frac{v / c}{\sqrt{1-\left(\frac{v}{c}\right)^{2}}}$ then for $c=1$, these equations can be written as

$$
\cosh \theta=\frac{1}{1-v^{2}} \equiv \gamma \& \sinh \theta=\frac{v}{1-v^{2}} \equiv v \gamma
$$

By computing (11)

$$
\begin{gathered}
y^{0}=\gamma\left(x^{0}-v x^{1}\right) \\
y^{1}=\gamma\left(x^{1}-v x^{0}\right) \\
\text { and } y^{2}=x^{2}, y^{3}=x^{3}
\end{gathered}
$$

For Galilean Transformation put $c=\infty$ such that $\gamma=1$, which implies from (9) \& (10) $y^{0}=x^{0} \& y^{1}=x^{1}-v x^{0}$. 


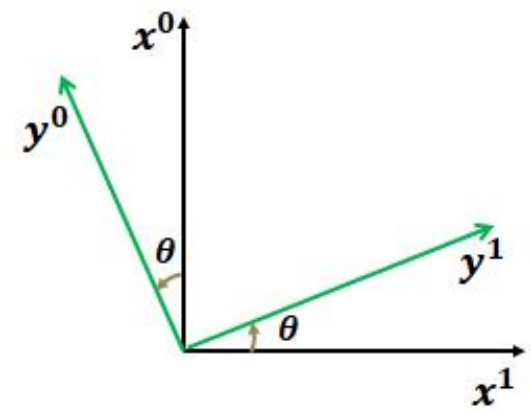

Figure 3: Lorentz rotation of coordinate is rotation by $\theta$

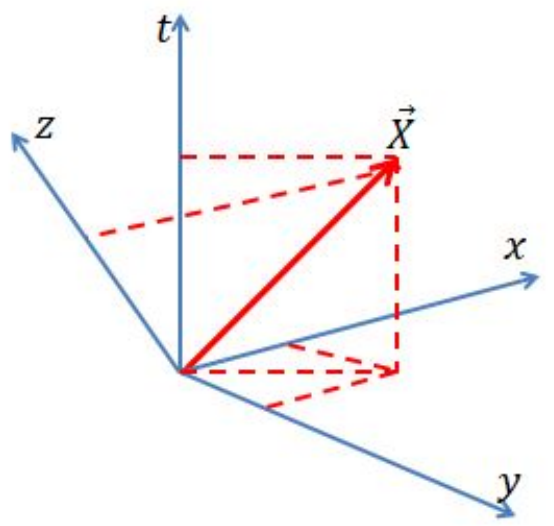

Figure 4: 4-position vector. ( $\mathrm{t}, \mathrm{x}, \mathrm{y}, \mathrm{z}$ ) are orthogonal to each other. (It is dummy image to represent $4 \mathrm{D}$ )

\subsubsection{Lorentz Rotation}

Rotation $\mathrm{x}$ by arbitrary angle theta to get $\mathrm{y}$.

$$
\left[\begin{array}{l}
y^{0} \\
y^{1} \\
y^{2} \\
y^{3}
\end{array}\right]=\left[\begin{array}{cccc}
\cos \theta & \sin \theta & 0 & 0 \\
-\sin \theta & \cos \theta & 0 & 0 \\
0 & 0 & 1 & 0 \\
0 & 0 & 0 & 1
\end{array}\right]\left[\begin{array}{l}
x^{0} \\
x^{1} \\
x^{2} \\
x^{3}
\end{array}\right]
$$

\subsection{4-vector}

\subsubsection{4-Position $(\vec{X})$}

$$
\begin{gathered}
\vec{X}=X^{t} \vec{e}_{t}+X^{x} \vec{e}_{x}+X^{y} \vec{e}_{y}+X^{z} \vec{e}_{z} \\
\vec{V}=X^{\mu} \vec{e}_{\mu} \\
X^{\mu}=\left[\begin{array}{l}
X^{t} \\
X^{x} \\
X^{y} \\
X^{z}
\end{array}\right]=\left[\begin{array}{l}
t \\
x \\
y \\
z
\end{array}\right]
\end{gathered}
$$


3.3.2 4-Velocity $\left(\vec{U}=\frac{\partial \vec{X}}{\partial \tau}\right)$

$$
\begin{gathered}
\vec{U}=\frac{\partial \vec{X}}{\partial \tau} \equiv \frac{\partial\left(X^{t} \vec{e}_{t}+X^{x} \vec{e}_{x}+X^{y} \vec{e}_{y}+X^{z} \vec{e}_{z}\right)}{\partial \tau} \\
\left\{\frac{\partial \vec{e}_{\mu}}{\partial \tau}=0\right\} \\
\Longrightarrow \vec{U}=\frac{\partial X^{t}}{\partial \tau} \vec{e}_{t}+\frac{X^{m}}{\partial \tau} \vec{e}_{m} \\
\left\{\text { from }(19): \frac{\partial X^{t}}{\partial \tau}=\gamma \equiv \frac{1}{\sqrt{1-v^{2}}}\right\} \\
\vec{U}=\gamma \vec{e}_{t}+\frac{X^{m}}{\partial X^{0}} \frac{X^{0}}{\partial \tau} \vec{e}_{m} \\
\vec{U}=\gamma\left(v^{0} \vec{e}_{t}+\frac{X^{m}}{\partial X^{0}} \vec{e}_{m}\right) \\
\vec{U}=\gamma v^{\mu} \vec{e}_{\mu}=U^{\mu} \vec{e}_{\mu}
\end{gathered}
$$

Inner product of 4 -velocity is

$$
U^{\mu} U_{\mu}=-1
$$

or explicitly (15) as $U^{\mu} U_{\mu}=-c^{2}$

\subsubsection{4-Momentum $(\vec{P}=m \vec{U})$}

$$
\vec{P}=m \vec{U}=\underbrace{m \gamma}_{\text {relativistic mass }} \vec{v}
$$

In component form

$$
P^{\mu}=m \gamma\left[\begin{array}{l}
v^{t} \\
v^{x} \\
v^{y} \\
v^{z}
\end{array}\right]
$$

Inner product of momentum $\eta(\vec{P}, \vec{P})=P^{\mu} P_{\mu}$ for metric

$$
\begin{gathered}
P^{\mu} \eta_{\mu \nu} P^{\nu}=m^{2}\left(U^{t} U_{t}-U^{n} U_{n}\right)=m\left(-\frac{1}{1-v^{2}}+\frac{v^{2}}{1-v^{2}}\right) \\
P^{\mu} P_{\mu}=-m^{2}
\end{gathered}
$$

To compute the relation between momentum and energy, let's take the proper time (4) explicitly

$$
\begin{aligned}
& d \tau=\sqrt{(d t)^{2}-\left(d x^{n}\right)^{2}} \\
& d \tau=d t \sqrt{1-\underbrace{\left(\frac{d x^{n}}{d t}\right)^{2}}_{v}}
\end{aligned}
$$




$$
\frac{d \tau}{d t}=\sqrt{1-v^{2}}
$$

Lagrangian can we written as

$$
\mathcal{L}=-m \sqrt{1-\dot{x}^{2}}
$$

where ' - ' is just convention. 3 -momentum is written as

$$
P_{n}=\frac{\partial \mathcal{L}}{\partial \dot{x}^{n}}=\frac{m \dot{x}_{n}}{\sqrt{1-\left(\dot{x}^{n}\right)^{2}}}
$$

From (16), $P_{\mu}=m U_{\mu}$, and the time component of 4-momentum is $P_{0}=m U_{0}$

Using $(20)$ and $(21)$, Hamiltonian $\left(H=\dot{x}^{n} P_{n}-\mathcal{L}\right)$ can be written as

$$
H=\frac{m}{\sqrt{1-v^{2}}}=m U_{0}
$$

Now, (18) can be re-written as where $P^{\mu} P_{\mu}=-m^{2}$ where negative sign before mass is convection as in Lagrangian then

$$
\begin{gathered}
P^{0} P_{0}-P^{1} P_{1}-P^{2} P_{2}-P^{3} P_{3}=-m^{2} \\
\left(m U^{0}\right)^{2}-\left(m U^{1}\right)^{2}-\left(m U^{2}\right)^{2}-\left(m U^{3}\right)^{2}=-m^{2}
\end{gathered}
$$

from $(22) \Longrightarrow m U^{0}=-E$

$$
-E^{2}+p^{2}=-m^{2}
$$

where $p$ is Lorentz invariant 3-momentum. Let's put speed of light (c) in (23), this gives $P^{0}=E / c=\sqrt{(m c)^{2}+p^{2}}$.

From $(22) P^{0}=m c \gamma$, let's use the binomial expansion $(1+y)^{-n}=1+n x+n(n+1) / 2$ ! for $\gamma=\left(1-v^{2}\right)^{-\frac{1}{2}}$ then $(22)$ can be written as $P^{0}=m c\left(1+\frac{1}{2} \frac{v^{2}}{c^{2}}+. .(\right.$ higher order terms $\left.)\right)$, let's multiply this expression by $c$

$$
c P^{0}=\underset{\text { restenergy }}{m c^{2}}+\underset{\text { non-relativistic kinetic energy }}{\frac{m v^{2}}{2}}+. .(\text { higher order terms })
$$

Similar expression get for spacial components of 4-momentum (21)

$$
P^{n}=m v^{2}\left(1+\frac{1}{2} \frac{v^{2}}{c^{2}}+. .(\text { higher order terms })\right)
$$

Now, relativistic energy can be obtained from (24) and (25)

$$
E^{2}=\left(m c^{2}\right)^{2}+(p c)^{2}
$$

Rest-energy of any particle (other than photon) is $E=m c^{2}$. For natural units where $c=1$, (23) is similar to relativistic energy.

For special case when $v=c=1, P^{0}=\frac{m}{\sqrt{1-1}} \Longrightarrow m=0$. Thus, for photons (mass-less particle) (26) is $E=p c$. And they travel on null geodesic $n_{\mu \nu} P^{\mu} P^{\nu}=0$. This gives further implications of particle being light-like, time-like or space-like.

$$
n_{\mu \nu} P^{\mu} P^{\nu}=\left\{\begin{array}{l}
-1, \text { time - like } \\
0, \text { light - like } \\
1, \text { space - like }
\end{array}\right.
$$




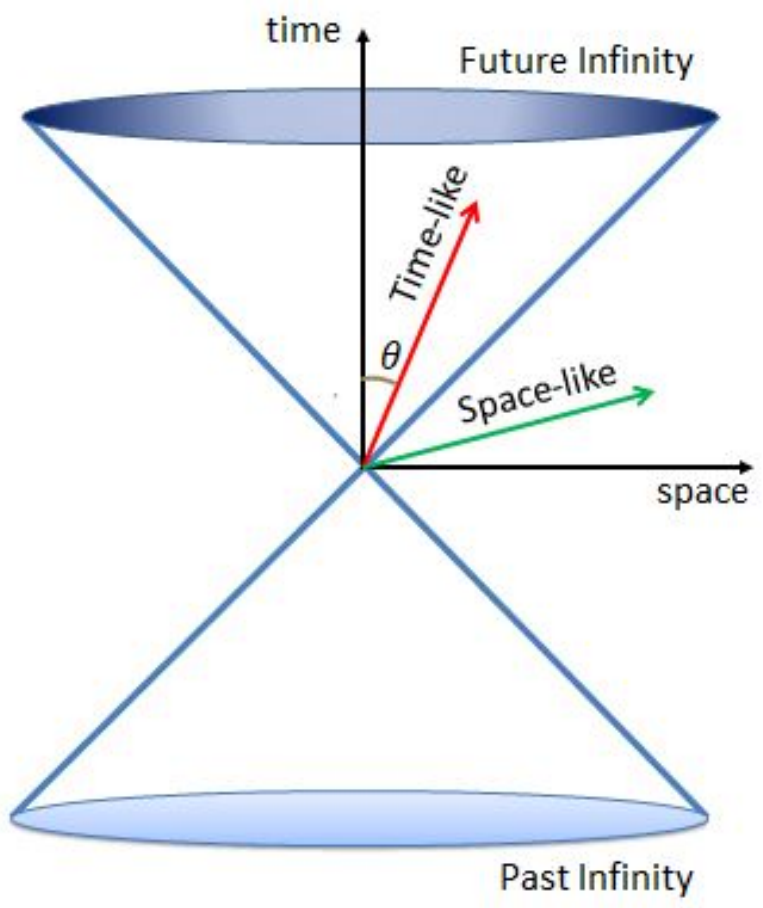

Figure 5: Light cone. Boundaries of light cone is null trajectories at $45^{\circ}$. Red worldline is time-like trajectory, and green woldline is space-like trajectory.

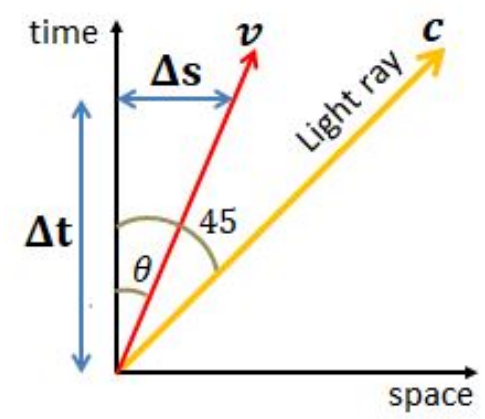

Figure 6: A particle travels with velocity $v$ and Lightray travels with velocity $c$. Speed of light is constant for local inertial frame shows that light rays move at $45^{\circ}$. Worldline of a particle moving at $v<c$ is always time-like

If we consider the proper time $\tau$ for photons (or (massless) light-like particles) then from equation (19) $d \tau=0$ this implies proper for photon is 0 .

From (27) Minkowski space-time can represented using a light-cone (figure 5), and the particle trajectories are called worldlines.

For time-like particle (Figure 6), $\tan \theta=\frac{\Delta s}{\Delta t}=\frac{1}{c}\left(\frac{\Delta x^{i}}{\Delta t}\right)^{2}=\frac{v}{c}$. For $v=c$, this equations gives $\theta=45^{\circ}$. Hence, postulate 1 implies that lightcone is absolute. In other words, all the light rays will move at $45^{\circ}$ irrespective of its origin for Minkowski space.

\subsubsection{4-Acceleration $\left(\vec{\chi}=\frac{d \vec{U}}{d \tau}\right)$}

In special relativity, 4-acceleration is uniform for the observer with respect to its proper time. This implies $\chi$ is function of $\tau$, i.e, $\chi(\tau)$.

4 -acceleration in components 


$$
\chi^{\mu}=\frac{d U^{\mu}}{d t} \equiv \gamma \frac{d}{d \tau}\left[\begin{array}{c}
c \\
v^{x} \\
v^{y} \\
v^{z}
\end{array}\right]
$$

Inner product of acceleration is

$$
\chi^{\mu} \chi_{\mu}=\eta_{\mu \nu} \chi^{\mu} \chi^{\nu}=k^{2}
$$

From (15) and (28) let's show the orthogonality to 4-velocity and 4-acceleration, i.e., their inner product must be zero.

$$
\begin{aligned}
\eta_{\mu \nu} U^{\mu} U^{\nu} & =-1 \\
2 \eta_{\mu \nu} \frac{d U^{\mu}}{d t} U^{\nu} & =0 \\
\Longrightarrow \quad \vec{\chi} \cdot \vec{U} & =0
\end{aligned}
$$

Now, (15) and (28) implies that (ignoring the y and z coordinates)

$$
\begin{gathered}
-1=-\left(\frac{d t}{d \tau}\right)^{2}+\left(\frac{d x}{d \tau}\right)^{2} \\
k^{2}=-\left(\frac{d^{2} t}{d \tau^{2}}\right)^{2}+\left(\frac{d^{2} x}{d \tau^{2}}\right)^{2}
\end{gathered}
$$

From (30) and (31)

$$
\frac{d t}{d \tau}=\cosh (\omega(\tau)) \& \frac{d x}{d \tau}=\sinh (\omega(\tau))
$$

Using (32) in (31) will give

$$
\begin{gathered}
k^{2}=\left(\omega^{\prime}(\tau)\right)^{2} \underbrace{\left(\cosh ^{2}(\omega(\tau))-\sinh ^{2}(\omega(\tau))\right)}_{=1} \\
k=\left(\omega^{\prime}(\tau)\right) \Longrightarrow k \tau=\omega
\end{gathered}
$$

Substituting (33) into (32), that is,

$$
\frac{d t}{d \tau}=\cosh (k \tau) \& \frac{d x}{d \tau}=\sinh (k \tau)
$$

gives

$$
\begin{aligned}
& t=\frac{1}{k} \sinh (k \tau)+t_{o} \\
& x=\frac{1}{k} \cosh (k \tau)+x_{o}
\end{aligned}
$$

From (34), hyperbolic curve is obtain

$$
-c^{2}\left(t-t_{o}\right)^{2}+\left(x-x_{o}\right)^{2}=\frac{c^{4}}{k^{2}}
$$




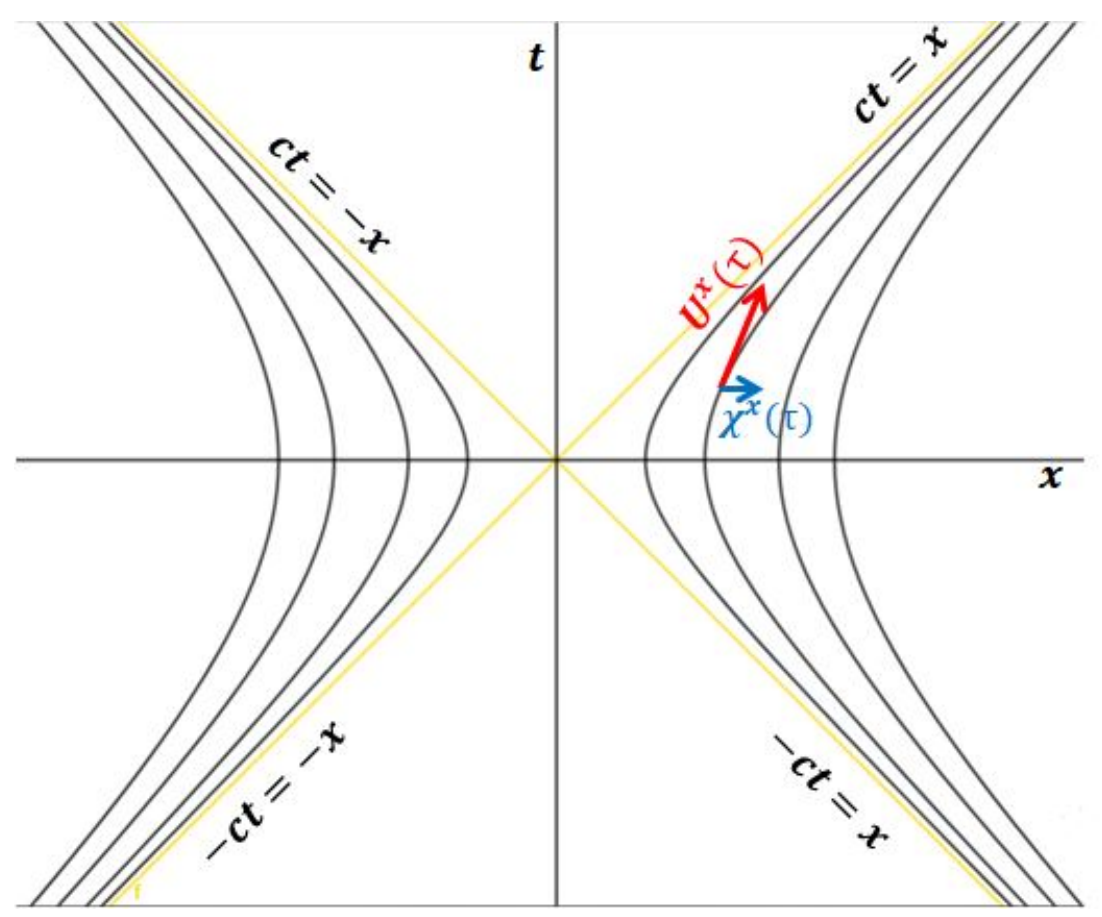

Figure 7: Equation (35) gives the family of hyperbolic curves for the observers experiencing uniform acceleration. It is called the Rindler coordinate, where $c t= \pm x$ acts as accelerated (or Rindler) horizon.

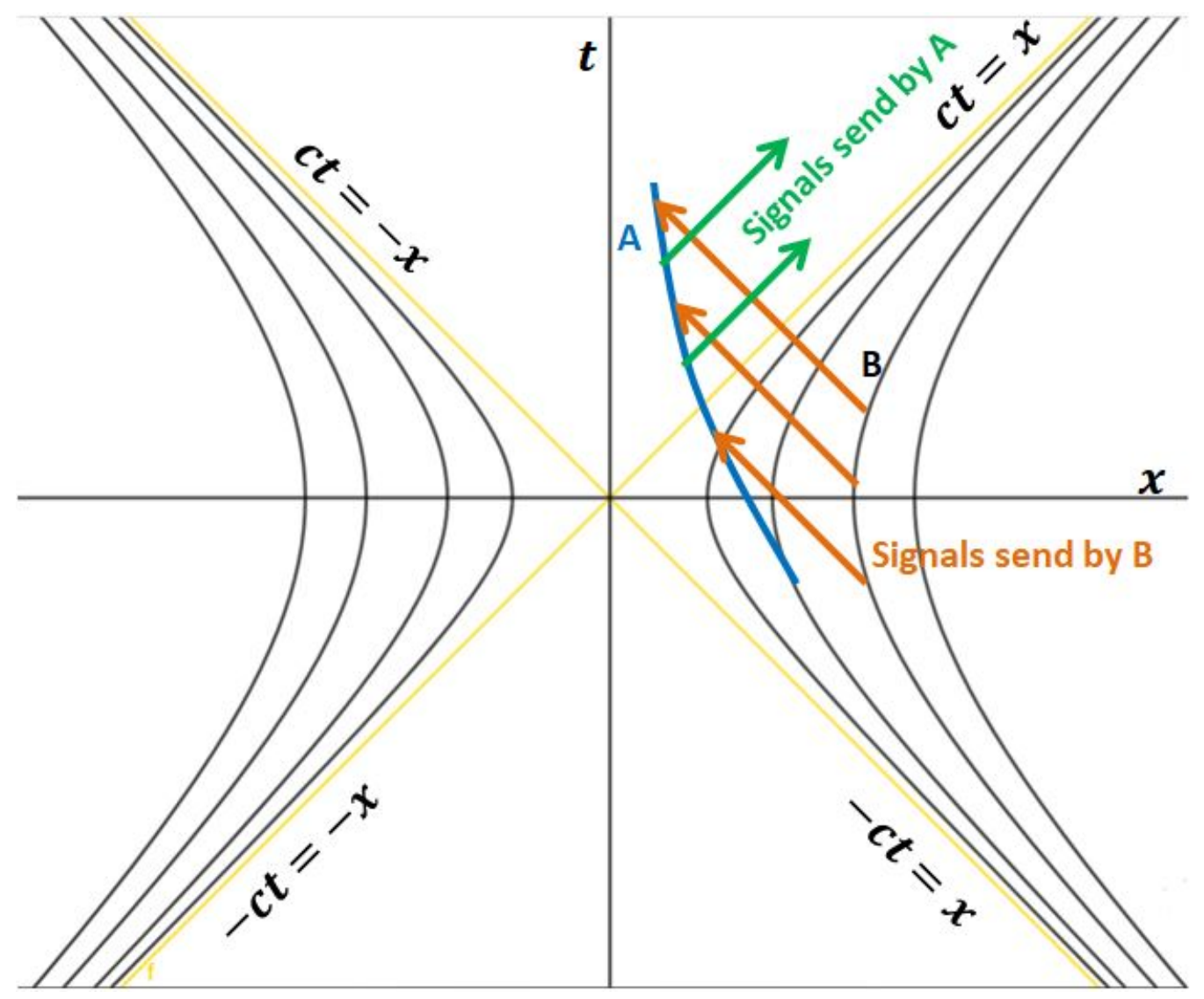

Figure 8: Observer A beyond the accelerated horizon can no longer send the signals back to B as light rays are only allowed to move at $45^{\circ}$. However, A can receive signals from B 


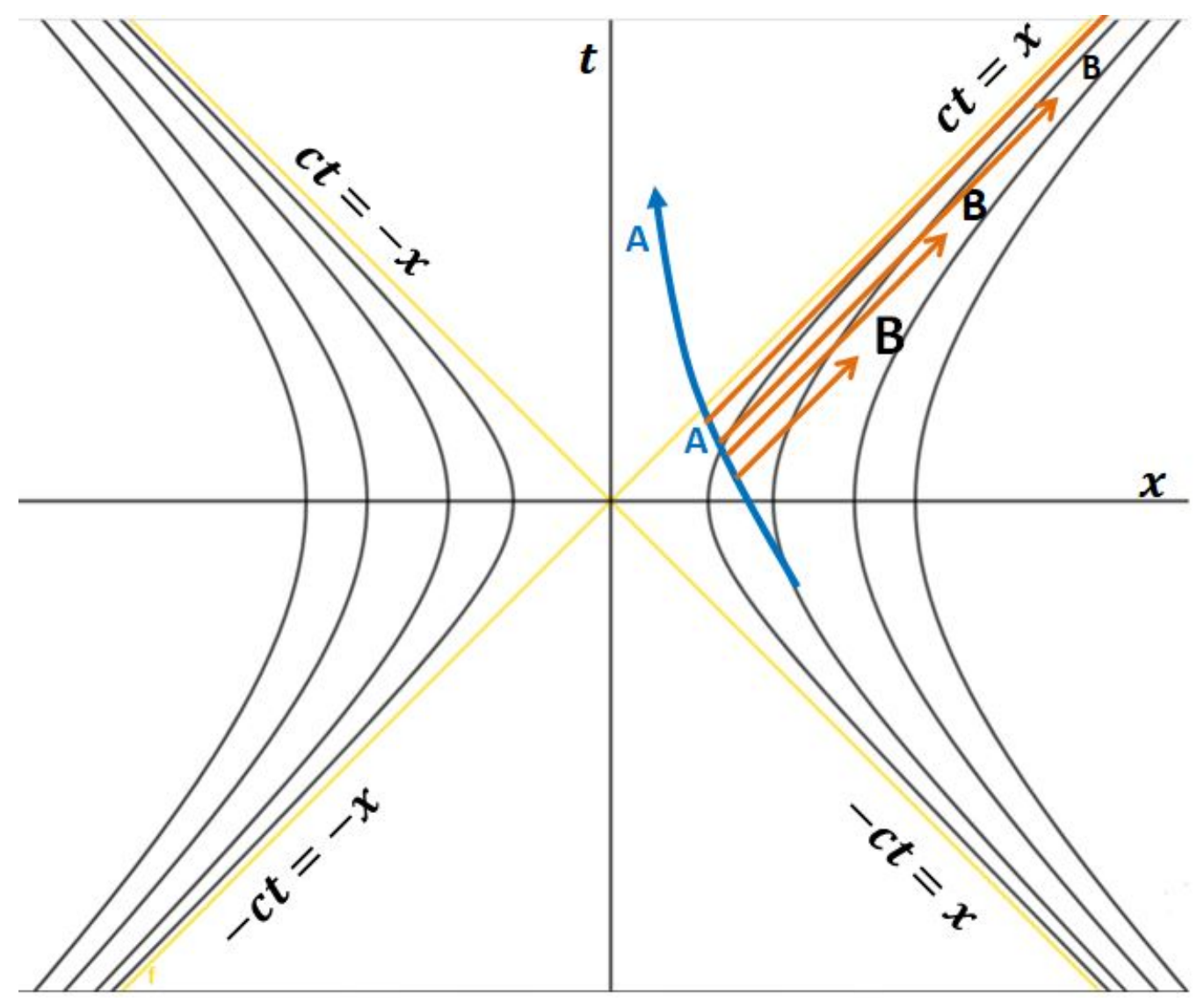

Figure 9: Observer $\mathrm{B}$ is receiving signals from $\mathrm{A}$, and due to the acceleration experienced by $\mathrm{B}$ with respect to $\mathrm{A}$ (at the horizon), these signals will keep getting redder as B will approach the future. Due to which A will observe B moving closer to the horizon but never pass it. However, from B's frame of reference B is already beyond the horizon.

\subsection{Rindler Coordinate}

Rindler coordinates are the coordinates made from hyperbolic worldlines $\left(X^{\mu}(\tau)\right)$ such that the proper time $(\tau)$ is spacetime invariant. These hyperbolic curves show the uniform acceleration experienced by the observer in Minkowski space. In general relativity, Einstein defines the Equivalence principle due which acceleration is the consequences curved manifold, that is, the observer following the hyperbola is experiencing gravity.

- Any observer in uniform acceleration will asymptotically approach the speed of light in the far infinity (either $+/$ - $^{-}$with respect to the non-local observer(s). Hence, as a consequence of relativity, any two non-local observers will see each other redshifted.

- Any observer, say A, who passed the accelerated horizon can no longer be visible to the outside observer B. Thus, one could always say A is moving faster than light with respect to an outside observer(s) B. A can receive signals from B but can't send the signals back to $\mathrm{B}$ without violating the constant speed of light. Refer figure 8.

- Any observer, say B, outside the accelerated horizon, can not see the A passing the horizon. B will see the A froze near the horizon, and as B is in uniform acceleration, A will keep on getting redderwhich $\mathrm{A}$ will perceive as B getting closer and closer to the horizon. At some asymptotic future, A will vanish (or get fainter) for B or outside observer. Refer figure 9.However, from B point of view, B will pass the horizon without any worry. (Complimentary) 
- Rindler coordinates can explain the basic geometry of black holes.

\section{Maxwell Theory}

Maxwell's equations were not consistent with Galilean relativity. It possessed the problem of the invariance principle until, in 1905, Einstein introduced the Lorentz transformation to describe the relativistic dynamics of a charged particle, hence, giving the invariant electromagnetic field. (Refer postulates 1 and 2)

\subsection{Relativistic Lorentz Force}

Let a charged particle moving on its worldline $X^{\mu}(\tau)$ such that the action is defined as

$$
\text { action }=\int\left(-m d \tau-e A_{\mu}\left(X^{\mu}(\tau)\right) d X^{\mu}\right)
$$

where $m$ is mass of the particle independent of $\tau, e$ is coupling constant of field (or charge of the particle) and $A_{\mu}$ is vector potential of electromagnetic field. Further modification on action can yield

$$
\begin{aligned}
\text { action } & =\int\left(-m \frac{d \tau}{d t}-e A_{\mu} \frac{d X^{\mu}}{d t}\right) d t \\
& =\int\left(-m \sqrt{1-\dot{x}^{2}}-e(A_{0} \underbrace{\frac{d X^{0}}{d t}}_{=1}+A_{n} \dot{X}^{n})\right) d t
\end{aligned}
$$

such that Lagrangian of field is

$$
\mathcal{L}=\underbrace{-m \sqrt{1-v^{2}}}_{L_{1}} \underbrace{-e\left(A_{0}+A_{n} \dot{X}^{n}\right)}_{L_{2}}
$$

Euler-Lagrange equation for (36) is

$$
\frac{d}{d t} \frac{\partial \mathcal{L}}{\partial \dot{X}^{\mu}}=\frac{\partial \mathcal{L}}{\partial X^{\mu}}
$$

and conjugate momentum $\frac{\partial \mathcal{L}}{\partial \dot{X}^{\mu}}$ for the $L_{1}=-m \sqrt{1-v^{2}}$ is usual 4-momentum $m U^{\mu}$.

$$
\text { LHS of } L_{1}=-m \frac{d U^{\mu}}{d t}
$$

Euler-Lagrange equation for (36) $L_{2}$ can be written as

$$
\frac{d}{d t} \frac{\partial \mathcal{L}}{\partial \dot{X}^{m}}=\frac{\partial \mathcal{L}}{\partial X^{m}}
$$

Using (36) $L_{2}$ in (38)

$$
\begin{aligned}
\frac{d}{d t}\left(-e A_{n}(t, X)\right) & \approx-e\left(\frac{\partial A_{0}}{\partial X^{n}}+\frac{\partial A_{j}}{\partial X^{n}} \dot{X}^{j}\right) \\
-e\left(\frac{\partial A_{n}}{\partial t}+\frac{\partial A_{n}}{\partial X^{j}} \frac{d X^{j}}{d t}\right) & \approx-e\left(\frac{\partial A_{0}}{\partial X^{n}}+\frac{\partial A_{j}}{\partial X^{n}} \dot{X}^{j}\right) \\
-e\left(\frac{\partial A_{n}}{\partial t}+\frac{\partial A_{n}}{\partial X^{j}} \dot{X}^{j}\right) & \approx-e\left(\frac{\partial A_{0}}{\partial X^{n}}+\frac{\partial A_{j}}{\partial X^{n}} \dot{X}^{j}\right)
\end{aligned}
$$


Using (37) and (39) let's write the whole Euler-Lagrange equation for Lagrangian (36)

$$
\begin{aligned}
m \eta_{\mu \alpha} \frac{d U^{\alpha}}{d t} & =-e\left(\frac{\partial A_{0}}{\partial X^{n}}+\frac{\partial A_{j}}{\partial X^{n}} \dot{X}^{j}\right)+e\left(\frac{\partial A_{n}}{\partial t}+\frac{\partial A_{n}}{\partial X^{j}} \dot{X}^{j}\right) \\
m \eta_{\mu \alpha} \frac{\partial U^{\alpha}}{\partial \tau} & =e\left(\left(\frac{\partial A_{n}}{\partial X^{0}}-\frac{\partial A_{0}}{\partial X^{n}}\right) U^{0}+\left(\frac{\partial A_{n}}{\partial X^{j}}-\frac{\partial A_{J}}{\partial X^{n}}\right) U^{j}\right) \\
m \eta_{\mu \alpha} \frac{d^{2} X^{\alpha}}{d \tau^{2}} & =\eta_{\mu \alpha} \frac{d P^{\mu}}{d \tau} \equiv e F_{\mu \nu} U^{\nu}
\end{aligned}
$$

where $F$ is Faraday tensor. And it is defined as follows $\left(F_{\mu \nu}=\eta_{\mu \alpha} F_{\nu}^{\alpha}\right)$

$$
F_{\mu \nu}=\partial_{\mu} A_{\nu}-\partial_{\nu} A_{\mu}
$$

In matrix notation (due to anti-symmetry diagonal terms are zero)

$$
F_{\mu \nu}=\left[\begin{array}{cccc}
0 & -E_{x} & -E_{y} & -E_{z} \\
E_{x} & 0 & B_{z} & -B y \\
E_{y} & -B_{z} & 0 & -B x \\
E_{z} & B_{y} & -B_{x} & 0
\end{array}\right]
$$

where $\vec{E}$ and $\vec{B}$ are electric and magnetic fields.

Let's deduce the Lorentz force 4-vector from (40)

$$
\vec{F}=m \frac{d^{2} \vec{X}}{d \tau^{2}}=\gamma e(\vec{E}+\vec{v} \times \vec{B})
$$

\subsection{Maxwell Equations}

Let's use (41) and (42) to derive first and second Maxwell equation.

Lagrangian of electromagnetic wave is $\mathcal{L}=-\frac{1}{4} F^{\mu \nu} F_{\mu \nu}$, and Euler-Lagrange for this Lagrangian is

$$
\begin{aligned}
& \partial_{\alpha}\left(\frac{\partial\left(-\frac{1}{4} F^{\mu \nu} F_{\mu \nu}\right)}{\partial_{\xi}\left(\partial_{\alpha} A_{\beta}\right)}\right)=\underbrace{\partial_{\beta} \mathcal{L}}_{=0} \\
& \Longrightarrow \partial_{\alpha}\left(\frac{\partial\left(-\frac{1}{4} F^{\mu \nu} F_{\mu \nu}\right)}{\partial_{\xi}\left(\partial_{\alpha} A_{\beta}\right)}\right)=0
\end{aligned}
$$

Using Leibniz rule in equation (44),

$$
-\frac{1}{4} \partial_{\alpha}\left(\frac{\partial F^{\mu \nu}}{\partial_{\xi}\left(\partial_{\alpha} A_{\beta}\right)} F_{\mu \nu}+F^{\mu \nu} \frac{\partial F_{\mu \nu}}{\partial_{\xi}\left(\partial_{\alpha} A_{\beta}\right)}\right)=0
$$

Re-writing $F^{\mu \nu}$ in 2-form (rank 2 covariant tensor) as

$$
F^{\mu \nu}=\eta^{\mu \sigma} \eta^{\nu \gamma} F_{\sigma \gamma}
$$

where $F_{\sigma \gamma}=\partial_{\sigma} A_{\gamma}-\partial_{\gamma} A_{\sigma}$.

then

$$
\begin{aligned}
\frac{\partial F_{\sigma \gamma}}{\partial_{\xi}\left(\partial_{\alpha} A_{\beta}\right)} & =\frac{\partial\left(\partial_{\sigma} A_{\gamma}-\partial_{\gamma} A_{\sigma}\right)}{\partial_{\xi}\left(\partial_{\alpha} A_{\beta}\right)} \\
& =\frac{\partial\left(\delta_{\sigma}^{\alpha} \delta_{\gamma}^{\beta} \partial_{\alpha} A_{\gamma}-\delta_{\gamma}^{\alpha} \delta_{\sigma}^{\beta} \partial_{\gamma} A_{\sigma}\right)}{\partial_{\xi}\left(\partial_{\alpha} A_{\beta}\right)} \\
& =\delta_{\sigma}^{\alpha} \delta_{\gamma}^{\beta}-\delta_{\gamma}^{\alpha} \delta_{\sigma}^{\beta}
\end{aligned}
$$


Using (46) in (45) then (44) can be written as

$$
\begin{aligned}
-\frac{1}{4} \partial_{\alpha}\left(F_{\mu \nu} \eta^{\nu \sigma} \eta^{\nu \gamma}\left(\delta_{\sigma}^{\alpha} \delta_{\gamma}^{\beta}-\delta_{\gamma}^{\alpha} \delta_{\sigma}^{\beta}\right)+F^{\mu \nu}\left(\delta_{\mu}^{\alpha} \delta_{\nu}^{\beta}-\delta_{\nu}^{\alpha} \delta_{\mu}^{\beta}\right)\right) & =0 \\
\underbrace{-\frac{1}{4} \partial_{\alpha}\left(F^{\alpha \beta}-F^{\beta \alpha}+F^{\alpha \beta}-F^{\beta \alpha}\right)}_{\text {this expression can be written in } 2-\text { form }} & =0 \\
\partial_{\alpha} F^{\alpha \beta} & =0
\end{aligned}
$$

this gives

$$
\partial^{\mu} F_{\mu \nu}=0
$$

For $\nu=t(47)$ will produce first Maxwell equation, that is,

$$
\underset{=0}{\partial^{t} F_{t t}}+\partial^{x} F_{x t}+\partial^{x} F_{y t}+\partial^{x} F_{z t}=0
$$

Above equation implies that divergence for electric field $\vec{V}$ is zero for free space, that is,

$$
\nabla \cdot \vec{E}=0
$$

first Maxwell equation For $\nu=x(47)$ gives

$$
\begin{aligned}
& \partial^{t} F_{t x}+\partial^{x} F_{x x}+ \partial^{x} F_{y x}+\partial^{x} F_{z x}=0 \\
& \Longrightarrow(\nabla \times B)_{x}=\frac{\partial E_{x}}{\partial t}
\end{aligned}
$$

and for $\nu=(x, y, z)(47)$, it will give second Maxwell equation, which implies that curl of the magnetic field $\vec{B}$ is equal time varying electric field $\vec{E}$

$$
\nabla \times \vec{B}=\frac{\partial \vec{E}}{\partial t}
$$

From (47) Faraday tensor can be written without indices,

$$
d F=0
$$

which implies $F=d A$ and $d^{2} A=0$. It gives a nice theorem which is as follows;

Theorem 1 Let $M$ be a manifold such that $d^{2}=0$, where $d$ is exterior derivative.

For the charge free space RHS of equation (47) is zero, however, For the charge-free space, the RHS of the equation (47) is zero. However, for a charged particle (in analogous to the original Maxwell equation), let's define a 3-form charge $J$ for which is (47) can be re-witten as

$$
\partial_{\mu} F^{\mu \nu}=J^{\nu}
$$

from equation $(51)$

$$
d^{*} F={ }^{*} J
$$

where ${ }^{*} F$ and ${ }^{*} J$ means dual of $F$ and dual of $J$. This equation (53) implies $d J=0$. In other words we can say that charge is conserved. $\partial_{\nu} J^{\nu}=0$. 
Maxwell equations (49) and (50) from (52) can be written as

$$
\nabla \cdot \vec{E}=\rho
$$

and

$$
\nabla \times \vec{B}-\frac{\partial \vec{E}}{\partial t}=\mathcal{J}
$$

where $\rho=J^{0}$ and $\mathcal{J}=J^{n}$

REMARK: Other two equations of Maxwell are the consequences of 1 , that is, $\partial_{\mu} B^{\mu}=0$.

Or using Bianchi Identity

$$
\partial_{\gamma} F^{\alpha \beta}+\partial_{\alpha} F^{\beta \gamma}+\partial_{\beta} F^{\gamma \alpha}=0
$$

which implies

$$
\nabla \cdot \vec{B}=0
$$

and if any index, say $\alpha$ in the Bianchi identity (56) is set to zero then it yields

$$
\nabla \times \vec{E}+\partial_{t} \vec{B}=0
$$

Comments: Maxwell theory is been explained in classical non-relativistic and relativistic classical and quantum theory. It suggests that charged particles in the Lorentz frame will create an electric and magnetic field, and in principle, electric and magnetic fields are different components of the Faraday tensor.

\section{General Relativity}

"Space-time tells matter how to move; matter tells space-time how to curve"-John Archibald Wheeler

Einstein 1905 work on special relativity, successfully combined electrodynamics and Galilean relativity. But it only worked for flat spacetime. In other words, it is used only for inertial frames. Later, Einstein wanted to combine Newtonian gravity with special relativity to obtain a unified theory for physics. In 1915, He derived the field equation for gravity purely from the physical aspect of the Gauss law of gravity. Though, David Hilbert solved the problem around five days before Einstein but gave the credit to Einstein for ethical reasons. Hilbert used the variational principle to obtain the field equation. His derivation was analytical and more elegant. (Refer [6])

\subsection{Equivalence Principle}

Weak Equivalence Principle: A free-falling particle will experience the same acceleration independent of the system, either at rest with respect to the uniform gravitational field or subjected to uniform acceleration with respect to the inertial system. And the converse is also true. 
Einstein Equivalence Principle: For an arbitrary local region $\nless$ defined on pseudoRiemannian Manifold $M$, any observer in that region will not detect the gravitational field. In other words, spacetime is locally flat such that the physic of special relativity is valid for $\Varangle$.

Strong Equivalence Principle: Physics is same for a rest observer under uniform gravitational field and for the uniform accelerated observer. Vaguely stating gravity and acceleration are equivalent.

Principle of general relativity: "All laws of Nature preserve their form under arbitrary coordinate changes."

Mach's principle: "The inertia of a body is determined by its relation to all other masses in the universe.."

\subsection{Definitions, Properties \& Theorems}

Geodesic: Geodesic is the shortest path between two events in spacetime for a defined metric.

Torsion-free property: For a manifold M, Lie bracket of the defined on the tangent space should be zero, that is, tangent vectors $\vec{v}$ and $\vec{u}$ along the flow curves must commute.

$$
[\vec{v}, \vec{u}]=0
$$

from above relation one can show that connections coefficients are symmetric for lower indices,

$$
\Gamma_{\mu \nu}^{\rho}=\Gamma_{\nu \mu}^{\rho}
$$

Metric compatibility property: For a manifold $M$, the length of the vectors along the flow curves do not change. This implies Lie derivative $\mathcal{L}$ of the metric $g$ for the Killing vector field $\vec{t}$ is zero, that is,

$$
\mathcal{L}_{\vec{t}} g_{\mu \nu}=0
$$

From (59) and (60) one can state the fundamental theorem of Riemannian geometry.

Theorem 2 The fundamental theorem of Riemannian geometry states that for a Riemannian manifold (or pseudo-Riemannian manifold) $M$, the connection is torsion-free, and $M$ obeys metric compatibility.

\subsection{Variation in determinant metric - det $g$ and volume element $\sqrt{-\operatorname{det} g}$}

In pseudo-Riemannian manifold, I will consider a general metric $g=g_{\mu \nu} d x^{\mu} d x^{\nu}=-1$.

By using the following identity of the linear algebra $\ln ($ det $P)=\operatorname{tr}(\ln P)$ where $Q \stackrel{\text { def }}{=} \ln P$, $\mathrm{P}$ and $\mathrm{Q}$ are square matrix with non-zero determinant. And variation of this identity gives

$$
(\operatorname{det} P)^{-1} \ln (\delta \operatorname{det} P)=\operatorname{tr}\left(P^{-1} \ln \delta P\right)
$$

comparing above result with metric $g$ yields,

$$
\delta \operatorname{det} g=\operatorname{det} g g^{\mu \nu} \delta g_{\mu \nu}
$$

Now, From (61) let's compute the variation in the volume element $\sqrt{-\operatorname{det} g}$.

$$
\delta \sqrt{-\operatorname{det} g}=\frac{-\delta g}{\sqrt{-\operatorname{det} g}}=\frac{-\operatorname{det} g g^{\mu \nu} \delta g_{\mu \nu}}{\sqrt{-\operatorname{det} g}}=\frac{1}{2} \sqrt{-\operatorname{det} g} g^{\mu \nu} \delta g_{\mu \nu}
$$

I will use the result (61) and (62) to obtain the Einstein flied equation in section 5.5 


\subsection{Geodesic equation of variational principle}

Let the test particle is moving on the curve $X^{\mu}(\tau)$ on the pseudo-Riemannian manifold $M$. Action $S$ is given by

$$
S=\int_{\tau_{1}}^{\tau_{2}} \underbrace{\sqrt{-g_{\mu \nu} U^{\nu} U^{\mu}}}_{\mathcal{L}} d \tau
$$

Let's vary the action $S$ by $\delta S$, keeping the ends fixed. This infinitesimal change in $S$ will produce the extremum path between endpoints if $\delta S=0$. This extremum path is called a geodesic curve.

$$
\delta S=-m \delta \int_{\tau_{1}}^{\tau_{2}} \sqrt{-g_{\mu \nu} U^{\nu} U^{\mu}} d \tau=0
$$

$\delta$ can introduced inside the integral, that is,

$$
\begin{aligned}
\delta S & =-m \int_{\tau_{1}}^{\tau_{2}} \delta \sqrt{-g_{\mu \nu} U^{\nu} U^{\mu}} d \tau \\
& =m \int_{\tau_{1}}^{\tau_{2}} \frac{\delta\left(g_{\mu \nu} U^{\nu} U^{\mu}\right)}{2 \sqrt{-g_{\mu \nu} U^{\nu} U^{\mu}}} d \tau \\
& =\frac{m}{2} \int_{\tau_{1}}^{\tau_{2}} \delta\left(g_{\mu \nu} U^{\nu} U^{\mu}\right) d \tau
\end{aligned}
$$

$\delta$ will on the $\left(g_{\mu \nu} U^{\nu} U^{\mu}\right)$ like product rule

$$
\begin{aligned}
\delta S & =\frac{m}{2} \int_{\tau_{1}}^{\tau_{2}}\left(\delta g_{\mu \nu} U^{\nu} U^{\mu}+g_{\mu \nu} \delta U^{\nu} U^{\mu}+g_{\mu \nu} U^{\nu} \delta U^{\mu}\right) d \tau \\
& =\frac{m}{2} \int_{\tau_{1}}^{\tau_{2}}\left(\delta g_{\mu \nu} U^{\nu} U^{\mu}+2 g_{\mu \nu} \delta U^{\nu} U^{\mu}\right) d \tau
\end{aligned}
$$

Variation in metric $g_{\mu \nu}$ can be interpreted as

$$
\delta g_{\mu \nu}=\partial_{\gamma} g_{\mu \nu} \delta X^{\gamma}
$$

And $\left(2 g_{\mu \nu} \delta U^{\nu} U^{\mu}\right)$ can be written as

$$
2 g_{\mu \nu} \delta U^{\nu} U^{\mu}=\frac{d}{d \tau}\left(2 g_{\mu \nu} \delta X^{\nu} U^{\mu}\right)-2 \frac{d g_{\mu \nu}}{d \tau} \delta X^{\nu} U^{\mu}-2 g_{\mu \nu} \delta X^{\nu} \frac{d U^{\mu}}{d \tau}
$$

Let's use (65) and (66) in $\delta S$ to get

$$
\begin{aligned}
\delta S & =\frac{m}{2} \int_{\tau_{1}}^{\tau_{2}}\left(\partial_{\gamma} g_{\mu \nu} \delta X^{\gamma} U^{\nu} U^{\mu}+\frac{d}{d \tau}\left(2 g_{\mu \nu} \delta X^{\nu} U^{\mu}\right)-2 \frac{d g_{\mu \nu}}{d \tau} \delta X^{\nu} U^{\mu}-2 g_{\mu \nu} \delta X^{\nu} \frac{d U^{\mu}}{d \tau}\right) d \tau \\
& =\frac{m}{2} \int_{\tau_{1}}^{\tau_{2}}\left(\partial_{\gamma} g_{\mu \nu} \delta X^{\gamma} U^{\nu} U^{\mu}-2 \frac{d g_{\mu \nu}}{d \tau} \delta X^{\nu} U^{\mu}-2 g_{\mu \nu} \delta X^{\nu} \chi^{\mu}\right) d \tau+\underbrace{\frac{m}{2} \int_{\tau_{1}}^{\tau_{2}} d \tau \frac{d}{d \tau}\left(2 g_{\mu \nu} \delta X^{\nu} U^{\mu}\right)}_{\text {after integration it is } 0} \\
& =\frac{m}{2} \int_{\tau_{1}}^{\tau_{2}}\left(\partial_{\nu} g_{\mu \gamma} U^{\gamma} U^{\mu}-2 \frac{d g_{\mu \nu}}{d \tau} U^{\mu}-2 g_{\mu \nu} \chi^{\mu}\right) \delta X^{\nu} d \tau \\
& =\frac{m}{2} \int_{\tau_{1}}^{\tau_{2}}(\partial_{\nu} g_{\mu \gamma} U^{\gamma} U^{\mu}-\underbrace{2 \partial_{\gamma} g_{\mu \nu}}_{\partial_{\gamma} g_{\nu \mu}+\partial_{\mu} g_{\nu \gamma}} U^{\gamma} U^{\mu}-2 g_{\mu \nu} \chi^{\mu}) \delta X^{\nu} d \tau \\
& =\frac{m}{2} \int_{\tau_{1}}^{\tau_{2}}\left(\partial_{\nu} g_{\mu \gamma} U^{\gamma} U^{\mu}-\partial_{\gamma} g_{\nu \mu} U^{\gamma} U^{\mu}-\partial_{\mu} g_{\nu \gamma} U^{\gamma} U^{\mu}-2 g_{\mu \nu} \chi^{\mu}\right) \delta X^{\nu} d \tau
\end{aligned}
$$


Now, let's re-write above expression as

$$
\delta S=m \int_{\tau_{1}}^{\tau_{2}}\left(\frac{1}{2}\left(\partial_{\nu} g_{\mu \gamma}-\partial_{\gamma} g_{\nu \mu}-\partial_{\mu} g_{\nu \gamma}\right) U^{\mu} U^{\gamma}-g_{\mu \nu} \chi^{\mu}\right) \delta X^{\nu} d \tau
$$

Using the (64) above equation gives the geodesic equation in metric format

$$
\chi^{\rho}=\frac{1}{2} g^{\mu \rho}\left(\partial_{\gamma} g_{\nu \mu}+\partial_{\mu} g_{\gamma \nu}-\partial_{\nu} g_{\mu \gamma}\right) U^{\mu} U^{\gamma}
$$

which can also be written using the Levi-Civita connection $\Gamma_{\mu \gamma}^{\rho}$ as

$$
\chi^{\rho}=\Gamma_{\mu \gamma}^{\rho} U^{\mu} U^{\gamma}
$$

Or explicitly

$$
\frac{d^{2} X^{\rho}}{d \tau^{2}}=\Gamma_{\mu \gamma}^{\rho} \frac{d X^{\mu}}{d \tau^{2}} \frac{d X^{\gamma}}{d \tau^{2}}
$$

where Levi-Civita connection

$$
\Gamma_{\mu \gamma}^{\rho}=\frac{1}{2} g^{\mu \rho}\left(\partial_{\gamma} g_{\nu \mu}+\partial_{\mu} g_{\gamma \nu}-\partial_{\nu} g_{\mu \gamma}\right)
$$

Comments: For a timelike geodesic, a particle will experience a longer proper time. Similarly, for spacelike geodesic, a particle will take a shorter proper length. And null geodesics are usual light trajectories. Hence, in general relativity, geodesics can explain time dilation and length contraction. There may exist more than one geodesic and curves for which particle may take longer proper time than timelike geodesic and shorter proper length for spacelike geodesic.

\subsection{Einstein Field Equation}

Let's take the scalar Lagrangian $R$ for which the Hilbert action (Einstein-Hilbert action) $S_{H}$ on spacetime (4D pseudo-Riemannian) manifold is

$$
S_{H}=\int d^{4} x \sqrt{-\operatorname{det} g} R
$$

where $R$ is Ricci Scalar (Trace of Ricci Curvature $R_{\mu \nu}$ ), i.e., $R=g^{\mu \nu} R_{\mu \nu}$ and $\sqrt{-\operatorname{det} g}$ is volume element (here, $g=g_{\mu \nu} d x^{\mu} d x^{\nu}$ ).

Hence, (71) can be re-written as

$$
S_{H}=\int d^{4} x \sqrt{-\operatorname{det} g} R_{\mu \nu} g^{\mu \nu}
$$

Varying the action $S_{H}(72)$ will give

$$
\begin{aligned}
\delta S_{H} & =\int d^{4} x \delta\left(\sqrt{-\operatorname{det} g} R_{\mu \nu} g^{\mu \nu}\right) \\
& =\int d^{4} x\left((\delta \sqrt{-\operatorname{det} g}) R_{\mu \nu} g^{\mu \nu}+\sqrt{-\operatorname{det} g}\left(\delta R_{\mu \nu}\right) g^{\mu \nu}+\sqrt{-\operatorname{det} g} R_{\mu \nu}\left(\delta g^{\mu \nu}\right)\right) \\
& =\int d^{4} x\left(-\frac{1}{2} \sqrt{-\operatorname{det} g} g_{\mu \nu} \delta g_{\mu \nu} R+\sqrt{-\operatorname{det} g} R_{\mu \nu} \delta g^{\mu \nu}+\sqrt{-\operatorname{det} g} g^{\mu \nu} \delta R_{\mu \nu}\right) \\
& =\int d^{4} x\left(\left(-\frac{1}{2} g_{\mu \nu} R+R_{\mu \nu}\right) \sqrt{-\operatorname{det} g} \delta g^{\mu \nu}+\sqrt{-\operatorname{det} g} g^{\mu \nu} \delta R_{\mu \nu}\right)
\end{aligned}
$$


Now, to compute the rest to the variation, let's first compute $\delta R_{\mu \nu}$.

Ricci curvature tensor in general can be defined as $R_{\mu \nu}=g^{\nu \alpha} R_{\mu \alpha \nu}^{\alpha}$, where $R_{\mu \alpha \nu}^{\alpha}$ is Riemann curvature tensor.

In tensor notation Riemann curvature tensor is written using covraiant derivative as $R(\vec{u}, \vec{v}) \vec{w}=$ $\left(\nabla_{\vec{u}} \nabla_{\vec{v}}-\nabla_{\vec{v}} \nabla_{\vec{u}}\right) \vec{w}$, which can be further computed in components;

$$
\begin{aligned}
u^{i} v^{j} w^{k} R\left(\vec{e}_{\alpha}, \vec{e}_{\beta}\right) \vec{e}_{\gamma} & =u^{\alpha} v^{\beta} v^{\gamma}\left(\nabla_{\alpha} \nabla_{\beta}-\nabla_{\beta} \nabla_{\alpha}\right) \vec{e}_{\gamma} \\
& =u^{\alpha} v^{\beta} v^{\gamma}\left(\nabla_{\alpha} \nabla_{\beta} \vec{e}_{\gamma}-\nabla_{\beta} \nabla_{\alpha} \vec{e}_{\gamma}\right) \\
& =u^{\alpha} v^{\beta} v^{\gamma}\left(\nabla_{\alpha} \Gamma_{\beta \gamma}^{\mu}-\nabla_{\beta} \Gamma_{\alpha \gamma}^{\mu}\right) \vec{e}_{\mu}
\end{aligned}
$$

And Ricci curvature is written as

$$
-R(\vec{u}, \vec{v}) \cdot \vec{v}=u^{\alpha} v^{\gamma} v_{\gamma}\left(\nabla_{\alpha} \nabla_{\gamma}-\nabla_{\gamma} \nabla_{\alpha}\right) e^{\gamma} \equiv u^{\alpha} v^{\gamma} v_{\gamma} \underbrace{\left(\nabla_{\alpha} \Gamma_{\gamma \mu}^{\gamma}-\nabla_{\gamma} \Gamma_{\alpha \mu}^{\gamma}\right)}_{-R_{\alpha \mu}} e^{\mu}
$$

Thus $\int d^{4} x \delta R_{\mu \nu}$ is

$$
\delta R_{\mu \nu}=\nabla_{\alpha} \delta \Gamma_{\mu \nu}^{\alpha}-\nabla_{\nu} \delta \Gamma_{\mu \alpha}^{\alpha}
$$

From (73) and (75), let's compute $\sqrt{-\operatorname{det} g} g^{\mu \nu} \delta R_{\mu \nu}$, that is, later terms of $\delta S_{H}$.

$$
\int d^{4} x \sqrt{-\operatorname{det} g} g^{\mu \nu} \delta R_{\mu \nu}=\int d^{4} x \sqrt{-\operatorname{det} g} g^{\mu \nu}\left(\nabla_{\alpha} \delta \Gamma_{\mu \nu}^{\alpha}-\nabla_{\nu} \delta \Gamma_{\mu \alpha}^{\alpha}\right)
$$

Using metric compatibility property (60), let's put $g^{\mu \nu}$ inside covariant derivative.

$$
\begin{aligned}
& \int d^{4} x \sqrt{-\operatorname{det} g} g^{\mu \nu} \delta R_{\mu \nu}=\int d^{4} x \sqrt{-\operatorname{det} g}\left(\begin{array}{ll}
\nabla_{\alpha} g^{\mu \nu} & \left.\delta \Gamma_{\mu \nu}^{\alpha}-\nabla_{\nu} g^{\mu \nu} \delta \Gamma_{\mu \alpha}^{\alpha}\right)
\end{array}\right. \\
& =\int d^{4} x \sqrt{-\operatorname{det} g}\left(\nabla_{\alpha} g^{\mu \nu} \delta \Gamma_{\mu \nu}^{\alpha}-\nabla_{\alpha} g^{\mu \alpha} \delta \Gamma_{\mu \alpha}^{\alpha}\right) \\
& =\int d^{4} x \sqrt{-\operatorname{det} g}\left(\nabla_{\alpha} g^{\mu \nu} \delta \Gamma_{\mu \nu}^{\alpha}-\nabla_{\alpha} g^{\mu \alpha} \delta \Gamma_{\mu \alpha}^{\alpha}\right)
\end{aligned}
$$

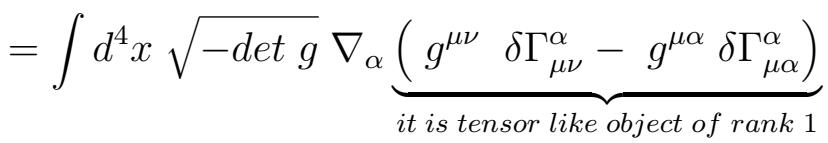

$$
\begin{aligned}
& =\int d^{4} x \sqrt{-\operatorname{det} g} \nabla_{\alpha} Z^{\alpha}
\end{aligned}
$$

Re-writing above expression

$$
\int d^{4} x \sqrt{-\operatorname{det} g} g^{\mu \nu} \delta R_{\mu \nu}=\int d^{4} x \sqrt{-\operatorname{det} g} \nabla_{\alpha} Z^{\alpha}
$$

Equation (76) is the divergence of $Z^{\alpha}$, this can be further computed using generalised Stokes theorem for a region $\Omega$ as

$$
\int_{\Omega} d \omega=\int_{\partial \Omega} \omega
$$

In other words, instead of computing the integral over region $\mathbb{R}^{4}$, it can be computed for the boundary of $\mathbb{R}^{4}$, that is, over $\mathbb{R}^{3}$.

For example, Stoke theorem for 3-vector is usual divergence theorem

$$
\int_{V} \nabla \cdot Z d^{3} x=\oint_{S} Z \cdot \hat{n} d^{2} x
$$


where $\mathrm{V}$ is 3 -volume region, $\mathrm{S}$ is surface (boundary of 3 -volume) of that region and $\hat{n}$ is normal vector to the surface $\mathrm{S}$

Similarly, for 4D spacetime manifold equation (76) can be reduced to boundary integral, i.e., the $3 \mathrm{D}$ boundary of spacetime manifold

$$
\int_{\Omega_{\mathbb{R}^{4}}} d^{4} x \sqrt{-\operatorname{det} g} \nabla_{\alpha} Z^{\alpha}=\int_{\partial \Omega_{\mathbb{R}^{3}}} d^{3} x \sqrt{-\operatorname{det} h} Z^{\alpha} n_{\alpha}
$$

where $\sqrt{-\operatorname{det} g}$ is volume element for boundary and $n_{\alpha}$ is normal component of the boundary.

Now, from the standard procedure of variation, the boundary terms are kept constant, which implies that the variation in the boundary must be zero. Hence, equation (77) is zero, $\int_{\mathbb{R}^{3}} d^{3} x \sqrt{-\operatorname{det} h} Z^{\alpha} n_{\alpha}=0$

$$
\Longrightarrow \int d^{4} x \sqrt{-\operatorname{det} g} g^{\mu \nu} \delta R_{\mu \nu} \equiv \int_{\mathbb{R}^{3}} d^{3} x \sqrt{-\operatorname{det} h} Z^{\alpha} n_{\alpha}=0
$$

Using the equation (78) in (73) and putting $\delta S_{H}=0$, that is,

$$
\delta S_{H}=\int d^{4} x\left(-\frac{1}{2} g_{\mu \nu} R+R_{\mu \nu}\right) \sqrt{-\operatorname{det} g} \delta g^{\mu \nu}=0
$$

which implies

$$
G_{\mu \nu}=R_{\mu \nu}-\frac{1}{2} g_{\mu \nu} R=0
$$

Einstein's Field equation for vacuum, where $G_{\mu \nu}$ is called Einstein Tensor.

\section{Matter Field}

Let the action $S_{M}$ be the action of matter field such that complete action $S$ is build from of matter action $S_{M}$ and Hilbert action $S_{H}$.

$$
S=S_{H}+\kappa S_{M}
$$

where $\kappa$ is constant.

Now, from (79) variation in action can be written as functional $\mathcal{L}$ which depends upon metric $g_{\mu \nu}$ such that

$$
\delta S=\int d^{4} x \frac{\delta \mathcal{L}}{\delta g^{\mu \nu}} \delta g^{\mu \nu}=0
$$

where $\mathcal{L}$ is just field Lagrangian.

Hence, equation (79) suggest

$$
\frac{1}{\sqrt{-\operatorname{det} g}} \frac{\delta S_{H}}{\delta g^{\mu \nu}}=\int d^{4} x G_{\mu \nu}=0
$$

Let's write complete action using (81) and (82), that is,

$$
\begin{gathered}
\frac{1}{\sqrt{-\operatorname{det} g}} \frac{\delta S}{\delta g^{\mu \nu}}=\frac{1}{2} \frac{1}{\sqrt{-\operatorname{det} g}} \frac{\delta S_{H}}{\delta g^{\mu \nu}}+\kappa \frac{1}{\sqrt{-\operatorname{det} g}} \frac{\delta S_{M}}{\delta g^{\mu \nu}}=0 \\
\frac{1}{2} \frac{1}{\sqrt{-\operatorname{det} g}} \frac{\delta S_{H}}{\delta g^{\mu \nu}}=\kappa \underbrace{\frac{-2}{\sqrt{-\operatorname{det} g} \frac{\delta S_{M}}{\delta g^{\mu \nu}}}}_{T_{\mu \nu}}
\end{gathered}
$$

which gives

$$
G_{\mu \nu}=\kappa T_{\mu \nu}
$$


where $T_{\mu \nu}$ is Energy-Momentum tensor, and $\kappa=\frac{8 \pi G}{c^{4}}$. For $c=1(80)$ is explicitly written as

$$
R_{\mu \nu}-\frac{1}{2} R g_{\mu \nu}=8 \pi G T_{\mu \nu}
$$

There are many ways to write Einstein field equation. However, in cosmology, there is an additional term called the cosmological constant $\Lambda$.

$$
R_{\mu \nu}-\frac{1}{2} R g_{\mu \nu}+\lambda g_{\mu \nu}=8 \pi G T_{\mu \nu}
$$

Under usual calculations cosmological constant is omitted, and write (85) is used because the value of cosmology constant is negligible in comparison of other terms, i.e., $10^{-52} \mathrm{~m}^{-2}$. Cosmology constant is used to calculate the dark energy or vacuum energy.

\section{Conservation of Energy}

Using the Bianchi identity (56) under covariant derivative in (85) implies $\nabla_{\nu} G^{\mu \nu}=\nabla_{\nu} T^{\mu \nu}=$ 0 , that is, divergence is zero. In other words, mass-energy is conserved.

Theorem 3 Lovelock's theorem 1 states that of any arbitrary pseudo-Riemannian manifold M, (0,2) symmetric tensor is Divergence free.(refer [4], [5])

Using Lovelock's theorem 1 3, equation (47) and equation (80) for vacuum electromagnetic field one can obtain the following result;

$$
R_{\mu \nu}-\frac{1}{2} R g_{\mu \nu}=\kappa^{\prime}\left(F_{\mu \sigma} F_{\nu}^{\sigma}-\frac{1}{4} g_{\mu \nu} F_{\sigma \rho} F^{\sigma \rho}\right)
$$

where $\kappa^{\prime}$ is constant.

Comments: Einstein's Field equation suggests that the geometry of spacetime is get influenced by mass or energy. In other words, the mass of an object deforms (curved) the spacetime such that any observer under-free will experience the acceleration, and the geodesic deviation will produce the tidal force (gravitational force). The geodesic deviation is further defined using the Ricci Curvature, which tracks the change in volume of an object along the geodesic in a curved manifold. Thus, the curvature is equivalent to mass which implies gravity.

\section{Summary}

In section 3, I have explained the introductory topics of special relativity and then derived the relativistic version of the Maxwell equation. I've also explained few points regarding the Rindler coordinate. Rindler coordinate is important to study the uniform acceleration in flat spacetime. In section 5, I have explained the geodesic equation, which suggests that any classical particle under free-fall will follow the geodesic curve. Later, I explained Einstein's field equation using the Hilbert action. Then with slight modification in the action, a complete field is obtained. 


\section{References}

[1] Charles W. Misner; Kip S. Thorne; John Archibald Wheeler (1973), Gravitation, W. H. Freeman,Princeton University Press, ISBN 0-7167-0344-0

[2] Carroll, Sean M. (2004), Spacetime and Geometry: An Introduction to General Relativity, San Francisco: Addison-Wesley, ISBN 978-0-8053-8732-2

[3] Hartle, James B. (2003), Gravity: an Introduction to Einstein's General Relativity, San Francisco: Addison-Wesley, ISBN 978-0-8053-8662-2

[4] Alberto Navarro, José Navarro, Lovelock's theorem revisited, Journal of Geometry and Physics, Volume 61, Issue 10, 2011, Pages 1950-1956, ISSN 0393-0440, https://doi.org/10.1016/j.geomphys.2011.05.004.

[5] Lovelock, D. (1971). The Einstein Tensor and Its Generalizations. Journal of Mathematical Physics, 12(3), 498-501. doi:10.1063/1.1665613

[6] Hilbert, D. (1915) Die Grundlagen der Physik (German original for free) (English translation for \$25), Konigl. Gesell. d. Wiss. Göttingen, Nachr. Math.-Phys. Kl. 395-407

[7] Susskind, L., \& Friedman, A. (2017). Special Relativity and Classical Field Theory: The Theoretical Minimum. Basic Books. 